\title{
Energy Band-Gap Engineering of Conjugated Microporous Polymers via Acidity-Dependent in Situ Cyclization
}

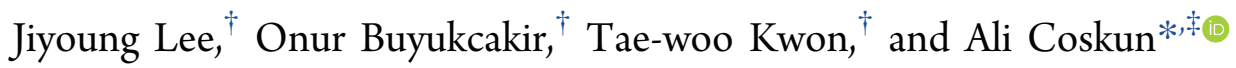 \\ ${ }^{\dagger}$ Graduate School of Energy, Environment, Water, and Sustainability (EEWS), Korea Advanced Institute of Science and Technology \\ (KAIST), Daejeon 34141, Republic of Korea \\ Department of Chemistry, University of Fribourg, Fribourg 1700, Switzerland
}

Supporting Information

\begin{abstract}
Conjugated microporous polymers (CMPs) offer a unique structure integrating $\pi$-conjugated backbone into a porous network for the simultaneous transport of charges and materials. However, tuning electronic properties of CMPs so far has been limited to an approach of varying the monomers, and the precious metal catalysts are inevitably needed for the $\mathrm{C}-\mathrm{C}$ coupling reaction. Here, we present a powerful strategy to synthesize CMPs and precisely tune their optical band gap and surface area through metal-free in situ cyclization reaction controlled by the acid strength of acid catalysts. Notably, the optical band gap of CMPs showed a linear relationship with the $\mathrm{p} K_{\mathrm{a}}$ of acid catalysts, which provides us with the ability to obtain the desired band gap between 2.07 and $3.35 \mathrm{eV}$, falling in the range of the visible solar spectrum. Moreover, CMPs exhibited excellent textural properties such as microporosity and high specific surface area.
\end{abstract}

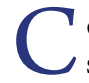
onjugated microporous polymers (CMPs), which is a subclass of porous organic polymers, are among the most promising materials for energy applications due to the unique combination of electron delocalization on the $\pi$-conjugated polymer backbone and microporous structure. ${ }^{1}$ A wide variety of methods to build porous structures with $\pi$-conjugation in two- and three-dimensional frameworks have been introduced using nonmetal- ${ }^{2}$ and metal- ${ }^{3}$ catalyzed polymerization strategies. The representative nonmetal-catalyzed polymers are covalent organic frameworks (COFs), pioneered by Yaghi and co-workers, ${ }^{4}$ that are constructed via reversible covalent bond formation such as imine ${ }^{5,6}$ and boroxine/boronate ester. ${ }^{7}$ However, though this reversible bond formation is critical for the crystallinity of these frameworks, it presents a fundamental challenge in their physicochemical stability under high temperature, humidity, and acidic/basic conditions. Some of these challenges have been nicely addressed by Banerjee and co-workers ${ }^{8,9}$ by engaging enol-to-keto tautomerization in locking the COF structure synthesized via Schiff base reaction. It is, however, important to note that while these strategies led to improved stabilities, the resulting COF structure lacks extended $\pi$-conjugation. On the other hand, the metalcatalyzed polymerization strategies under the kinetically controlled reaction conditions could enable the formation of conjugated, amorphous porous organic polymers with robust
$\mathrm{C}-\mathrm{C}$ bonds. ${ }^{10-12}$ However, the use of metal catalysts is problematic because of the high cost, pore blocking, and pore collapse during the extensive washing steps. In this regard, new metal-free polymerization strategies are highly desirable to construct porous organic polymers with strong $\mathrm{C}-\mathrm{C}$ linkages and a conjugated backbone.

Meanwhile, considering the growing interest for the utilization of CMPs in the context of heterogeneous photocatalysis, the systematic control over the electronic properties of CMPs has been a long-sought goal because each energy application requires different optimum band gap values: water splitting $\left(E_{\mathrm{g}}=1.5-2.5 \mathrm{eV}\right),{ }^{13,14} \mathrm{CO}_{2}$ reduction $\left(E_{\mathrm{g}}=1.75-3.0\right.$ $\mathrm{eV}),{ }^{15}$ and photovoltaic solar systems $\left(E_{\mathrm{g}}=1.65-3.27 \mathrm{eV}\right.$ for full visible-light absorption). ${ }^{16}$ Several efforts have been made to tune the band gap of CMPs by incorporating electron donor/acceptor groups and/or higher aromaticity into the monomers. ${ }^{3,15,17-19}$ However, the key constraints of these previous approaches are not only that the syntheses of the monomers require multistep reactions but also the quantitative relationship between the band gap of CMPs and the monomer structures has not been well-defined considering the differences in the extent of polymerization for each monomer.

Herein, we present a facile, metal-free synthetic strategy for the synthesis of CMPs, which involves a Schiff base reaction followed by an acid-catalyzed in situ cyclization reaction. This approach enabled us to precisely tailor the optical band gap of CMPs during the polymerization reaction, in which the extent of conjugation can be tuned through controllable in situ $\mathrm{C}-\mathrm{C}$ cyclization reaction by the acid strength of acid catalysts. Interestingly, the optical band gap and $\mathrm{p} K_{\mathrm{a}}$ of acid catalysts showed a linearly quantitative correlation, allowing us to predict and control the band gap of the resulting CMP.

From the design perspective, we identified 1,5,9-triaminetriphenylene (TATP) as the ideal core not only for the Schiff base reaction but also the fact that it provides the ideal molecular arrangement for the subsequent acid-catalyzed in situ cyclization. The two types of CMPs, uncyclized (ucCMP) and its cyclized analogue (cCMP), were synthesized (Figure 1a) by a solvothermal reaction of 1,5,9-triamine-triphenylene (TATP) and terephthalaldehyde (TPA) in a mixture of $N, N$ dimethylformamide/1,4-dioxane (10:1 v/v) and $3 \mathrm{M}$ aqueous acetic acid $(\mathrm{AcOH})$ and triflic acid $(\mathrm{TfOH})$, respectively. The reaction is suggested to proceed through two sequential steps; 

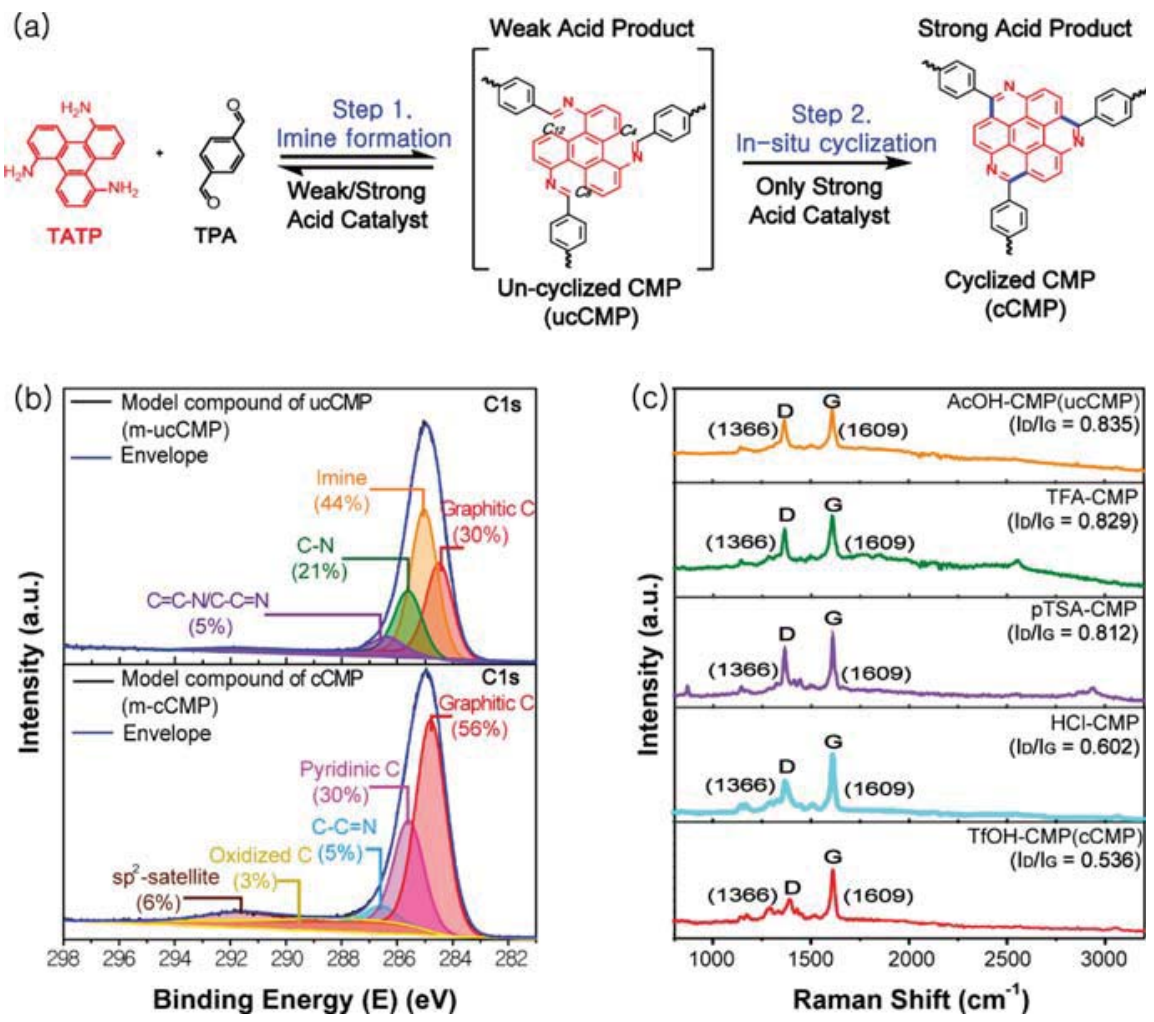

Figure 1. Synthesis and spectroscopic characterization of ucCMP and cCMP. (a) The syntheses of ucCMP and cCMP were achieved via Schiff base formation irrespective of acid strength and subsequent acidity-dependent in situ cyclization reactions. (b) XPS spectra of C 1s for m-ucCMP (top) and m-cCMP (bottom). (c) FT-Raman spectra of the series of CMPs measured at $1064 \mathrm{~nm}$ on a powder sample.

the first step involves the formation of the reversible imine linkages via the condensation reaction between amines of TATP and terephthalaldehyde irrespective of the acid strength, forming ucCMPs. In the second step, the in situ cyclization of ucCMP is only triggered by strong acids, analogous to the Pictet-Spengler cyclization reaction, ${ }^{20-22}$ through the irreversible dehydrogenation reaction of the imine carbons and the neighboring 4,8,12-positioned carbon atoms of TATP (denoted as $\mathrm{C}_{4}, \mathrm{C}_{8}$, and $\mathrm{C}_{12}$ in Figure 1a), yielding cCMPs. It should be noted that the second step became accelerated by the involvement of dicationic iminium ions which are favorably formed when the acidity of catalyst is increased. Moreover, Shudo and co-workers reported previously that weak acids can only promote to the formation of monocationic iminium ion, thus lowering conversion yields in the Pictet-Spengler cyclization reaction. ${ }^{20-22}$ According to this trend, partially cyclized CMPs were synthesized by using moderately strong acid catalysts, namely, trifluoroacetic acid (TFA, $\mathrm{p} K_{\mathrm{a}}=0.23$ ), $p$-toluene sulfonic acid ( $\left.\mathrm{pTSA}, \mathrm{p} K_{\mathrm{a}}=-2.80\right)$, and hydrogen chloride $\left(\mathrm{HCl}, \mathrm{p} K_{\mathrm{a}}=-6.30\right)$, between $\mathrm{AcOH}\left(\mathrm{p} K_{\mathrm{a}}=4.76\right)$ and $\mathrm{TfOH}\left(\mathrm{p} K_{\mathrm{a}}=-14.7\right)$. The CMPs are referred to as XCMP where $\mathrm{X}$ represents the acid catalyst. We also synthesized the model compounds of ucCMP (m-ucCMP) and cCMP (mcCMP) by reacting TATP and benzaldehyde under the same conditions using acetic acid $(\mathrm{AcOH})$ and triflic acid $(\mathrm{TfOH})$, respectively. ${ }^{23}$ The ucCMPs and cCMPs were found to be insoluble in common organic solvents. Additionally, the particle size distribution analysis revealed the formation of relatively smaller particles for cCMP compared to ucCMP (Figure S4).

The chemical structures of the CMP series were verified by using Fourier transform infrared (FT-IR) spectroscopy
(Figures S5-S7). The disappearance of amine stretching bands $\left(3300-3400 \mathrm{~cm}^{-1}\right)$ coupled with the presence of imine stretching bands $\left(1611 \mathrm{~cm}^{-1}\right)$ was clearly observed, thus indicating the successful formation of polymer network for all CMPs. The FT-IR spectrum of cCMP showed weaker $\mathrm{C}-\mathrm{H}$ $\left(2990-2866 \mathrm{~cm}^{-1}\right)$ and stronger $\mathrm{C}-\mathrm{C}\left(1440 \mathrm{~cm}^{-1}\right)$ stretching bands as well as stronger aromatic $\mathrm{C}-\mathrm{H}$ out of plane vibration bands $\left(888,825 \mathrm{~cm}^{-1}\right)$ compared to those of ucCMP, which are ascribed to the removal of hydrogens and to the formation of new $\mathrm{C}-\mathrm{C}$ bonds upon cyclization in good agreement with their corresponding model compounds. Notably, the appearance of the new peak at $165 \mathrm{ppm}$ in the ${ }^{13} \mathrm{C} \mathrm{CP} / \mathrm{MAS}$ NMR spectrum of cCMP also proves the evident structural changes upon cyclization (Figures S3). Powder X-ray diffraction (PXRD) analysis of CMPs revealed (Figure S7) their amorphous nature. X-ray photoelectron spectroscopy (XPS) analysis was carried out to confirm the in situ cyclization under strongly acidic conditions. The $\mathrm{C} 1 \mathrm{~s}$ spectrum of m-cCMP and m-ucCMP (Figure 1b) were deconvoluted into GaussianLorentzian shapes, where all peaks were assigned by the difference of electron density between atoms. ${ }^{24}$ The intensity of graphitic C peak for m-cCMP (56\%) is approximately two times higher than that for m-ucCMP (30\%), which originates from the increased number of $\mathrm{C}-\mathrm{C}$ bonds upon cyclization (Figure $1 \mathrm{~b}$ ). Importantly, the presence of $\mathrm{sp}^{2}$ satellite peak in $\mathrm{m}$-cCMP is attributed to the $\pi-\pi^{*}$ transition of electrons during X-ray irradiation, representing a strong evidence for the formation of extended $\pi$-conjugation upon cyclization. ${ }^{25}$ Importantly, the XPS spectra of ucCMP and cCMP showed a good accordance with those of the model compounds. The intensity of $\mathrm{sp}^{2}$ satellite peaks of ucCMP was found to be lower than that of $\mathrm{CCMP}$, supporting that $\mathrm{CCMP}$ has a higher degree 
of $\pi$-conjugation originating from the in situ cyclization. Importantly, the intensities of graphitic $\mathrm{C}$ and $\mathrm{sp}^{2}$ satellite peaks for CMP series were increased gradually with stronger acids, hence proving that the degree of both cyclization and $\pi$ conjugation are increased when the acidity of catalyst is increased (Figure S10-S16).

The graphitization of polymer networks before and after in situ cyclization was also examined by the Fourier TransformRaman spectroscopy (FT-Raman). As illustrated in Figure 1c, protonated Schiff base vibration band at $1657 \mathrm{~cm}^{-126}$ was not observed while the evident characteristic graphene D and G bands at 1366 and $1609 \mathrm{~cm}^{-1}$, respectively, were found in the Raman spectra of all CMPs. The intensity ratio of D and G bands $\left(I_{\mathrm{D}} / I_{\mathrm{G}}\right)$ indicating the graphitic nature of polymer networks was calculated to be $0.835,0.829,0.812,0.602$, and 0.536 for AcOH-, TFA-, pTSA-, $\mathrm{HCl}-$, and TfOH-CMP, respectively. The decreasing trend in $I_{\mathrm{D}} / I_{\mathrm{G}}$ ratio with increasing acid strength indicated the formation of higher number of $\mathrm{sp}^{2}$-hybridized graphitic carbon atoms via in situ cyclization. The $I_{\mathrm{D}} / I_{\mathrm{G}}$ ratio of 0.536 is comparable to those of the high-quality reduced graphene oxides (RGO), ${ }^{27}$ demonstrating that the cCMP has a well-developed 2D-framework structure. Additionally, we measured electrical conductivity for cCMP and ucCMP using a two-probe method (Figures S18). The electric current of cCMP and ucCMP were obtained as 7.5 and $3.7 \mathrm{pA}$ at $2.0 \mathrm{~V}$ voltage bias, respectively, thus showing the increased degree of $\pi$-conjugation due to cyclization. The optical band gaps of CMPs were comprehensively assessed by the solid-state UV/vis absorption spectroscopy. As expected, $\mathrm{UV} /$ vis absorption spectra (Figure 2a) of CMPs revealed a redshift with increasing acidity owing to the higher $\pi$ conjugation. Their optical band gaps $\left(E_{\mathrm{g}}\right)$ were calculated by the Tauc's equation, giving optical band gaps of 3.35, 3.21, 2.85, 2.55, and $2.07 \mathrm{eV}$ for AcOH-, TFA-, pTSA-, HCl-, and
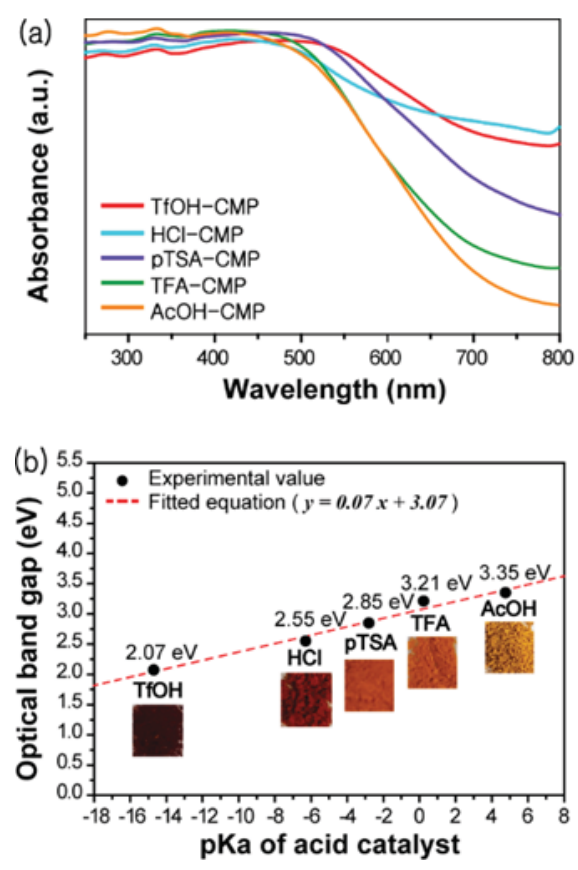

Figure 2. (a) Solid-state UV/vis spectroscopy analysis of CMPs. (b) Linear correlation between the optical band gap of CMPs and $\mathrm{p} K_{\mathrm{a}}$ of acid catalysts. The insets are photographic images of each CMP powder.
TfOH-CMP, respectively (Figure S19). The darker color of the resultant powders going from $\mathrm{AcOH}$ - to $\mathrm{TfOH}-\mathrm{CMP}$ also supported the decreased optical gaps derived from extended $\pi$ conjugation (Figure $2 \mathrm{~b}$, insets). Notably, we observed a linear correlation, $y=0.07 x+3.07$, between the optical band gap $(y)$ and $\mathrm{p} K_{\mathrm{a}}$ of acids $(x)$ (Figure $2 \mathrm{~b}$ ), where the optical band gap was decreased with an increase in the acid strength. Thus, any value of the optical band gap on the graph can be obtained by choosing the corresponding $\mathrm{p} K_{\mathrm{a}}$ of acid catalysts. Remarkably, complex synthetic designs and/or incorporation several different monomers are not needed for the precise band gap tuning.

The porosity of the CMPs was examined by argon adsorption-desorption isotherms at $87 \mathrm{~K}$. AcOH-CMP exhibited very low porosity (Figure 3a), whereas the other
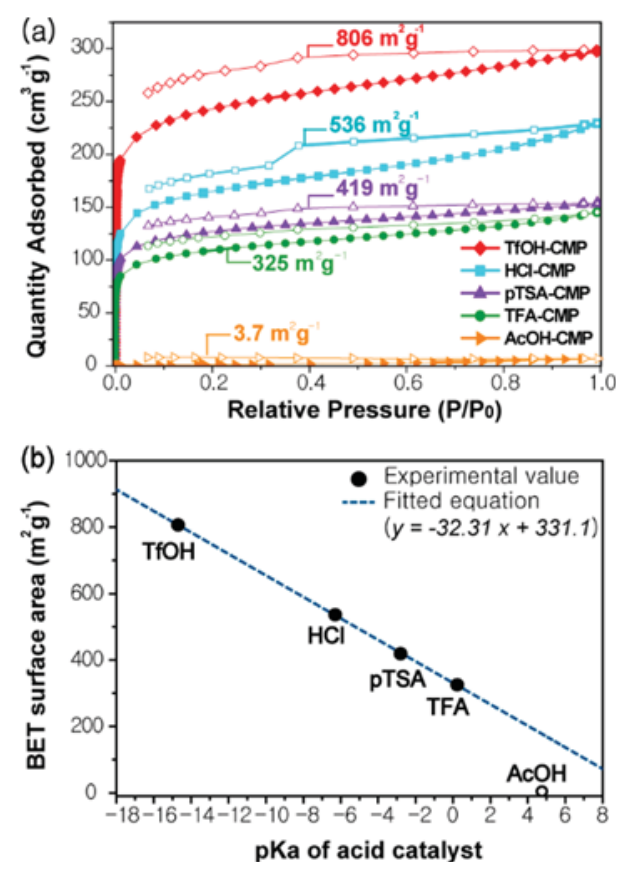

Figure 3. (a) Argon adsorption-desorption isotherms of CMPs at 87 $\mathrm{K}$. The closed symbols and open symbols represent adsorption and desorption, respectively. (b) Linear correlation between the BET surface area of CMPs and $\mathrm{p} K_{\mathrm{a}}$ of acid catalysts.

four CMPs exhibited a typical type-I isotherms, indicating the presence of the well-developed microporous structure. Interestingly, we also observed a correlation between the surface areas of CMPs and the strength of the acid catalyst. The higher surface areas were observed with increasing acid strength: AcOH-CMP $\left(3.7 \mathrm{~m}^{2} \mathrm{~g}^{-1}\right)<$ TFA-CMP $\left(325 \mathrm{~m}^{2} \mathrm{~g}^{-1}\right)$ $<$ pTSA-CMP $\left(419 \mathrm{~m}^{2} \mathrm{~g}^{-1}\right)<\operatorname{HCl}-\mathrm{CMP}\left(536 \mathrm{~m}^{2} \mathrm{~g}^{-1}\right)<$ TfOH-CMP $\left(806 \mathrm{~m}^{2} \mathrm{~g}^{-1}\right)$. Importantly, the surface area of CMPs correlates well with the $\mathrm{p} K_{\mathrm{a}}$ of acid catalysts (Figure $3 \mathrm{~b}$ ), where all spots of the surface area are perfectly located on the linear line except the AcOH-CMP. We hypothesized that the tilted geometry of uncyclized imine linkages blocks the accessibility of pores, but the cyclization gradually opens the pores owing to the planarization of the imine linkages in the form of aromatic rings, that is, the decreased flexibility and rotational freedom of organic linkers during cyclization resulted in increased accessibility of pores. ${ }^{3}$ We also carried out pore size distribution analysis of CMPs by using nonlocal density functional theory (NLDFT). As speculated, the 
average pore diameters increased going from TFA-CMP to $\mathrm{TfOH}-\mathrm{CMP}$, and the micropore volume was also increased from 0.15 for TFA-CMP to $0.32 \mathrm{~cm}^{3} \mathrm{~g}^{-1}$ for TfOH-CMP (Figure S21). This result clearly shows the impact of in situ cyclization on the textural properties of resulting CMPs

In conclusion, we presented a simple strategy to precisely tune the band gap and porosity of CMPs through controllable in situ cyclization by acid catalysts. We corroborated a strong linear correlation of acid strength with the optical band gap and surface area of CMPs. Based on this correlation, the optical band gap and surface area of CMPs can be fine-tuned in a quantitative fashion. Notably, this strategy eliminates the need for the precious metal catalysts for the $\mathrm{C}-\mathrm{C}$ bond formation and the multistep reactions for tuning the electronic properties of monomers and the resulting polymers. We not only report a powerful tool for controlling the electronic and textural properties of porous organic polymers but also present the potential of CMPs as organic semiconductors for energy and environmental applications.

\section{ASSOCIATED CONTENT}

\section{Supporting Information}

The Supporting Information is available

Full experimental methods, additional spectroscopic data, and references (PDF)

\section{AUTHOR INFORMATION}

\section{Corresponding Author}

*ali.coskun@unifr.ch

\section{ORCID}

Ali Coskun: 0000-0002-4760-1546

Notes

The authors declare no competing financial interest.

\section{ACKNOWLEDGMENTS}

This research was supported by the National Research Foundation of Korea (NRF) Grant funded by the Korea government (MEST) (2017R1A2B4004634).

\section{REFERENCES}

(1) Xu, Y.; Jin, S.; Xu, H.; Nagai, A.; Jiang, D. Chem. Soc. Rev. 2013, 42, 8012-8031.

(2) Jin, Y.; Hu, Y.; Zhang, W. Nat. Rev. Chem. 2017, 1, 0056.

(3) Chaoui, N.; Trunk, M.; Dawson, R.; Schmidt, J.; Thomas, A. Chem. Soc. Rev. 2017, 46, 3302-3321.

(4) Côté, A. P.; Benin, A. I.; Ockwig, N. W.; O’Keeffe, M.; Matzger, A. J.; Yaghi, O. M. Science 2005, 310, 1166-1170.

(5) Sun, Q.; Aguila, B.; Perman, J.; Earl, L. D.; Abney, C. W.; Cheng, Y.; Wei, H.; Nguyen, N.; Wojtas, L.; Ma, S. J. Am. Chem. Soc. 2017, 139, 2786-2793.

(6) Bai, L.; Gao, Q.; Zhao, Y. J. Mater. Chem. A 2016, 4, 1410614110

(7) Doonan, C. J.; Tranchemontagne, D. J.; Glover, T. G.; Hunt, J. R.; Yaghi, O. M. Nat. Chem. 2010, 2, 235.

(8) Kandambeth, S.; Mallick, A.; Lukose, B.; Mane, M. V.; Heine, T.; Banerjee, R. J. Am. Chem. Soc. 2012, 134, 19524-19527.

(9) Biswal, B. P.; Chandra, S.; Kandambeth, S.; Lukose, B.; Heine, T.; Banerjee, R. J. Am. Chem. Soc. 2013, 135, 5328-5331.

(10) Das, S.; Heasman, P.; Ben, T.; Qiu, S. Chem. Rev. 2017, 117, $1515-1563$

(11) Tan, L.; Tan, B. Chem. Soc. Rev. 2017, 46, 3322-3356.
(12) Buyukcakir, O.; Je, S. H.; Choi, D. S.; Talapaneni, S. N.; Seo, Y.; Jung, Y.; Polychronopoulou, K.; Coskun, A. Chem. Commun. 2016, 52, 934-937.

(13) Vyas, V. S.; Lau, V. W.-h.; Lotsch, B. V. Chem. Mater. 2016, 28, 5191-5204.

(14) Zhang, G.; Lan, Z. A.; Wang, X. Angew. Chem., Int. Ed. 2016 , $55,15712-15727$

(15) Sun, Z.; Talreja, N.; Tao, H.; Texter, J.; Muhler, M.; Strunk, J.; Chen, J. Angew. Chem., Int. Ed. 2018, 57, 7610-7627.

(16) Bildirir, H.; Gregoriou, V. G.; Avgeropoulos, A.; Scherf, U.; Chochos, C. L. Mater. Horiz. 2017, 4, 546-556.

(17) Schwarz, D.; Kochergin, Y. S.; Acharjya, A.; Ichangi, A.; Opanasenko, M. V.; Čejka, J.; Lappan, U.; Arki, P.; He, J.; Schmidt, J.; Nachtigall, P.; Thomas, A.; Tarábek, J.; Bojdys, M. J. Chem. - Eur. J. 2017, 23, 13023-13027.

(18) Ren, S.; Dawson, R.; Adams, D. J.; Cooper, A. I. Polym. Chem. 2013, 4, 5585-5590.

(19) Bi, S.; Lan, Z. A.; Paasch, S.; Zhang, W.; He, Y.; Zhang, C.; Liu, F.; Wu, D.; Zhuang, X.; Brunner, E.; Wang, X.; Zhang, F. Adv. Funct. Mater. 2017, 27, 1703146.

(20) Pictet, A.; Spengler, T. Ber. Dtsch. Chem. Ges. 1911, 44, 20302036.

(21) Ohwada, T.; Suzuki, T.; Shudo, K. J. Am. Chem. Soc. 1998, 120, 4629-4637.

(22) Yokoyama, A.; Ohwada, T.; Shudo, K. J. Org. Chem. 1999, 64, 611-617.

(23) Wei, J.; Han, B.; Guo, Q.; Shi, X.; Wang, W.; Wei, N. Angew. Chem., Int. Ed. 2010, 49, 8209-8213.

(24) Sánchez-López, J. C.; Donnet, C.; Lefèbvre, F.; FernándezRamos, C.; Fernández, A. J. Appl. Phys. 2001, 90, 675-681.

(25) Jackson, S. T.; Nuzzo, R. G. Appl. Surf. Sci. 1995, 90, 195-203.

(26) Eyring, G.; Mathies, R. Proc. Natl. Acad. Sci. U. S. A. 1979, 76, $33-37$.

(27) Eigler, S.; Enzelberger-Heim, M.; Grimm, S.; Hofmann, P.; Kroener, W.; Geworski, A.; Dotzer, C.; Röckert, M.; Xiao, J.; Papp, C.; Lytken, O.; Steinrück, H. P.; Müller, P.; Hirsch, A. Adv. Mater. 2013, 25, 3583-3587. 